\title{
Split-based computation of majority-rule supertrees
}

Anne Kupczok ${ }^{1,2}$

\begin{abstract}
Background: Supertree methods combine overlapping input trees into a larger supertree. Here, I consider splitbased supertree methods that first extract the split information of the input trees and subsequently combine this split information into a phylogeny. Well known split-based supertree methods are matrix representation with parsimony and matrix representation with compatibility. Combining input trees on the same taxon set, as in the consensus setting, is a well-studied task and it is thus desirable to generalize consensus methods to supertree methods.

Results: Here, three variants of majority-rule (MR) supertrees that generalize majority-rule consensus trees are investigated. I provide simple formulas for computing the respective score for bifurcating input- and supertrees. These score computations, together with a heuristic tree search minmizing the scores, were implemented in the python program PluMiST (Plus- and Minus SuperTrees) available from http://www.cibiv.at/software/plumist. The different MR methods were tested by simulation and on real data sets. The search heuristic was successful in combining compatible input trees. When combining incompatible input trees, especially one variant, MR(-) supertrees, performed well.

Conclusions: The presented framework allows for an efficient score computation of three majority-rule supertree variants and input trees. I combined the score computation with a heuristic search over the supertree space. The implementation was tested by simulation and on real data sets and showed promising results. Especially the MR(-) variant seems to be a reasonable score for supertree reconstruction. Generalizing these computations to multifurcating trees is an open problem, which may be tackled using this framework.
\end{abstract}

\section{Background}

Supertree methods amalgamate trees containing information from different, but overlapping, relationships into a larger supertree (e.g., [1]). The input trees need not have the same taxon sets, but the supertree contains all of the taxa present in at least one of the input trees. With this property, supertrees are applied to combine information present in different gene trees to infer relationships about larger sets of taxa (e.g., [2-6]).

Supertree methods can be distinguished by the elementary relationships they extract from the gene trees. These relationships can be splits (e.g., [7-9]), rooted triplets (e.g., [10-12]) or quartets (e.g., [13,14]). Here, I

Correspondence: anne.kupczok@ist.ac.at

${ }^{1}$ Center for Integrative Bioinformatics Vienna, Max F. Perutz Laboratories, University of Vienna, Medical University of Vienna, University of Veterinary

Medicine Vienna, Dr. Bohr-Gasse 9, A-1030 Vienna, Austria

Full list of author information is available at the end of the article focus on split-based supertree methods. A split is a bipartition of the taxa and a split of a tree corresponds to an edge in the tree that divides these two sets. Splits are compatible if they can occur together in a tree. Otherwise they are incompatible. A subsplit of a supertree split is generated by deleting some taxa from the taxon set. Thus an input tree split may be subsplit of a supertree split. The definition of compatibility can also be applied to splits on overlapping taxon sets, then the splits are first reduced to the common taxa and subsequently tested for compatibility.

It is natural for split-based supertree methods to first extract the splits from the input trees and code them into a matrix representation (see e.g., the splits from $\mathcal{G}$ in Table 1). The most widely applied supertree method is matrix representation with parsimony (MRP, $[7,8]$ ). In this approach, the matrix representation is interpreted as a binary alignment and the supertree is the most 
Table 1 Matrix representation and relationship matrix

\begin{tabular}{|c|c|c|c|c|c|c|c|}
\hline \multicolumn{8}{|c|}{ Matrix representation } \\
\hline & & \multicolumn{3}{|c|}{$\mathcal{S}$} & \multicolumn{3}{|c|}{$\mathcal{G}$} \\
\hline & $s_{1}$ & $s_{2}$ & $s_{3}$ & $s_{4}$ & $s_{5}$ & $g_{1}$ & $g_{2}$ \\
\hline A & 1 & 1 & 1 & 0 & 0 & - & - \\
\hline B & 1 & 1 & 1 & 0 & 0 & - & - \\
\hline C & 0 & 1 & 1 & 0 & 0 & 0 & 0 \\
\hline D & 0 & 0 & 1 & 0 & 0 & 1 & 0 \\
\hline E & 0 & 0 & 0 & 0 & 0 & - & - \\
\hline $\mathrm{F}$ & 0 & 0 & 0 & 1 & 0 & 1 & 0 \\
\hline G & 0 & 0 & 0 & 1 & 1 & 0 & 1 \\
\hline $\mathrm{H}$ & 0 & 0 & 0 & 1 & 1 & 0 & 1 \\
\hline \multicolumn{8}{|c|}{ Relationship matrix } \\
\hline & $s_{1}$ & $s_{2}$ & $s_{3}$ & $s_{4}$ & $s_{5}$ & $c_{i}$ & \\
\hline$g_{1}$ & c & c & i & i & c & 1 & \\
\hline$g_{2}$ & $c$ & $c$ & $c$ & C & $s$ & 0 & \\
\hline$b_{j}$ & 0 & 0 & 1 & 1 & 0 & & \\
\hline
\end{tabular}

Coding of the example trees in Figure 1. Only inner splits are shown.

parsimonious tree given the alignment. Matrix representation with compatibility (MRC, $[9,15]$ ) searches for the supertree maximizing the number of input splits that are a subsplit of a supertree split. MRC can also be understood as the compatibility method [16] applied to the binary alignment.

The task of summarizing trees on the same taxon set, the so called consensus setting, is well studied (e.g., $[17,18])$. Supertree methods can be understood as generalizations of consensus methods, that is, when applying a supertree algorithm in the consensus setting, the result should then be equivalent to the consensus. One popular consensus method is the majority-rule (MR) consensus, which produces a consensus tree that contains all splits present in at least half of the input trees. The MR consensus tree is a median tree under the RobinsonFoulds distance, in that, it is the tree with the smallest sum of the Robinson-Foulds distances to the input trees [19]. The Robinson-Foulds (RF) distance of two trees is the number of splits occurring in each of the trees, but are not found in the other [20].

Study of the consensus setting may also lead to important insights for supertree methods. Obviously, if a supertree method does not fulfill a property in the consensus setting, the property does not hold in general. For example, Wilkinson et al. [21] studied Pareto properties. They show that most supertree methods, including MRC and MRP, are Pareto on splits, i.e. the supertree contains a split if it is contained in all input trees. Methods are not co-Pareto on splits, if the supertree contains splits not supported by any input tree. E.g. MRC is co-Pareto on splits, but MRP is not. Some supertree methods show a bias in tree shape [22]. MRP shows a bias towards unbalanced shapes which is caused by the asymmetry of the underlying distance [23].

MRC and MRP can also be seen as median methods based on an asymmetric distance. The underlying distances are asymmetric since they only evaluate the fit of the input trees on the supertree and not vice versa. $\mathrm{MRC}$ is a median tree under the asymmetric RF distance, that is, the number of splits that are in the input tree but not in the pruned supertree. Thus, it generalizes the asymmetric median consensus [24,22]. Analogously, MRP can be interpreted as a median method based on the asymmetric parsimony distance.

Due to the asymmetric distances, MRC and MRP may favor relationships contradicting a majority of the input trees [25]. Cotton and Wilkinson [26] define majority-rule supertree methods as supertree methods generalizing the MR consensus. Different variants of MR supertrees exist and have been investigated. The main division among variants is between $\mathrm{MR}(-)$ and $\mathrm{MR}(+)$. The first evaluates distances between the pruned supertree and each input tree, while the second evaluates distances between the supertree and extended input trees. Here, extension refers to a method that adds missing taxa onto the input trees. Since this extension can be defined in multiple ways, multiple variants of $\mathrm{MR}(+)$ supertrees exist [26,27]. MR(+)s supertrees, a variant of $\mathrm{MR}(+)$ supertrees, can be solved exactly with an integer linear programming formulation [28]. MR (-) supertrees are closely related to RF supertrees [29] since both evaluate the RF distances between the pruned supertree and the input trees. Conceptually, there is a large difference between RF supertrees and MR supertrees. The aim of the first is to find at least one bifurcating tree of optimal score [29]. The approach of the latter, in contrast, is to find all trees of optimal score and to summarize them using the strict consensus method into a potentially multifurcating supertree. This also allows for labelling the MR supertree with values of support in the gene trees. By definition, finding all trees of optimal score also includes searching over multifurcating trees. Here a first attempt to solve the problem is performed that only searches for bifurcating trees of optimal score. From now on, I will also call these bifurcating trees of optimal score supertrees, since they contain all taxa from the input trees. They should not be confused with the MR supertrees that are obtained after the consensus step and can thus be multifurcating (see next section).

To date, there is only one study comparing the properties of different MR supertree variants [27] and I am not aware of any study on the performance of different MR supertree variants. The aim of this paper is to suggest a general framework for the distance computations underlying the MR supertree methods, to present an implementation evaluating different distance variants, and to compare these by simulation. 


\section{Results and Discussion Algorithm \\ Score computation}

I present the computation of majority-rule (MR) supertrees based on bifurcating and unrooted input- and supertrees. These trees contain only nodes with either one adjacent edge (the terminal nodes labeled with a taxon and the adjacent edge is a terminal edge) or with three adjacent edges (the inner nodes; non-terminal edges are also called inner edges). If an inner node of a tree has more than three adjacent edges, the tree is multifurcating. Note that bifurcating trees of $n$ taxa have $n$ 3 inner edges and, in comparison, multifurcating trees of $n$ taxa have fewer edges.

The Robinson-Foulds (RF) distance is only defined for trees labeled with the same taxa. There are two main ways to compute the RF distance between a supertree and an input tree when the input tree may contain only a subset of the taxa [26]:

1. Prune the supertree to the set of taxa in the input tree and compare the resulting tree to the input tree. This distance is called $d^{-}$.

2. Graft the remaining taxa in all possible ways onto the input tree, compute all distances, and take the minimal distance. There are different variants of grafting taxa onto input trees [27]. Here, two methods are investigated: (1) $d^{+}$extends an input tree to all bifurcating trees by placing additional taxa onto edges only and by resolving multifurcations in the input trees; (2) $d_{g}^{+}$extends an input tree to bifurcating and multifurcating trees by placing taxa onto edges or nodes, but does not resolve multifurcations present in the input tree.

Example distance computations are shown in Figure 1. The algorithm to compute the distances proceeds as follows. The matrix representation is a binary coding of the splits in the input- and supertree (Table 1). Next, a relationship matrix is computed from this matrix representation. For each (input split, supertree split) pair, the relationship matrix has three possible entries: subsplit; compatible but no subsplit; or incompatible. The relationship matrix of the example trees is given in Table 1. From this matrix, it is easy to see, whether the $i$-th input split is incompatible to at least one supertree split, then a binary variable $c_{i}$ is set to 1 . Analogously, a binary variable $b_{j}$ is set to 1 , if the $j$-th supertree split is incompatible to any input split. For a bifurcating input tree $\mathcal{G}$ and supertree $\mathcal{S}$, with $n_{\mathcal{G}}$ and $n$ taxa, respectively, the distance computations can then be simplified as follows (see methods section):

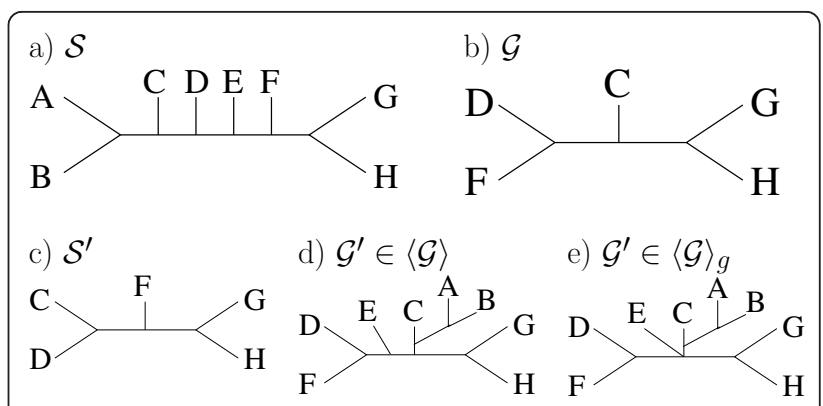

Figure 1 Example input- and supertree. Example supertree (a) and input tree (b) for distance computations. Both trees are identified by its split sets: $\mathcal{S}=\{A B|C D E F G H, A B C| D E F G H, A B C D \mid E F G H$, $F G H|A B C D E, G H| A B C D E F\}$ and $\mathcal{G}=\{D F|C G H, G H| C D F\}$ The supertree pruned to the taxa in $\mathcal{G}$ is shown in (c). Thus $d^{-}=2$. (d) shows a tree that results in an optimal distance of $d^{+}=4$ and (e) shows a tree that results in an optimal distance of $d_{g}^{+}=3$.

$$
\begin{aligned}
d^{-}(\mathcal{S}, \mathcal{G}) & =2 \times \sum_{i}^{n_{\mathcal{G}}-3} c_{i} \\
d^{+}(\mathcal{S}, \mathcal{G}) & =2 \times \sum_{j}^{n-3} b_{j}, \\
d_{g}^{+}(\mathcal{S}, \mathcal{G}) & =\sum_{i}^{n_{\mathcal{G}}-3} c_{i}+\sum_{j}^{n-3} b_{j} .
\end{aligned}
$$

The sum over the distances of all input trees $\mathcal{G} \in \mathcal{P}$ is called the score of a supertree with the respective supertree method, i.e., the score of $\mathcal{S}$ with $\operatorname{MR}(-)$ is $\sum_{\mathcal{G} \in \mathcal{P}} d^{-}(\mathcal{S}, \mathcal{G})$, with $\operatorname{MR}(+)$ it is $\sum_{\mathcal{G} \in \mathcal{P}} d^{+}(\mathcal{S}, \mathcal{G})$, and with $\operatorname{MR}(+) \mathrm{g}$ it is $\sum_{\mathcal{G} \in \mathcal{P}} d_{g}^{+}(\mathcal{S}, \mathcal{G})$. Note that the score of $\operatorname{MR}(-)$ also applies to multifurcating input trees (see methods section).

Here, $d^{+}$and $d_{g}^{+}$differ only by the way the taxa are placed because of the restriction to bifurcating input trees. When computing $d^{+}$, taxa can only be placed onto edges, and when computing $d_{g}^{+}$taxa can be placed onto edges or nodes. Note that $\mathrm{MR}(+)$ does not generalize majority-rule consensus but rather another consensus method called majority-rule $(+)$ consensus $[28,18]$. Here, I deal only with bifurcating input trees. It is easy to see that $\mathrm{MR}(+)$ supertrees also generalize MR consensus in this case: For bifurcating input trees on the same taxon set, $\mathrm{MR}(+)$ cannot place missing taxa or resolve multifurcations and thus $\mathrm{MR}(+)$ directly minimizes the $\mathrm{RF}$ distance to the input trees. Therefore the distinction between majority-rule consensus and majority-rule $(+)$ consensus is only important for non-bifurcating input trees.

Although these respective consensus methods behave differently in the general case [27], they are equivalent 
for bifurcating input trees, and I will treat all three methods, $\mathrm{MR}(-)$, MR(+)g, and $\operatorname{MR}(+)$ as supertree methods generalizing MR consensus for the remainder of the paper.

\section{Heuristic algorithm}

A heuristic search is necessary to finds upertrees with the minimal score. The three scores and a heuristic to search for supertrees with the minimal score were implemented in the python program PluMiST (Plus and Minus SuperTrees, available from http://www.cibiv.at/ software/plumist). The program takes bifurcating trees as input in the case of $\mathrm{MR}(+)$ and $\mathrm{MR}(+) \mathrm{g}$, and arbitrary trees in the case of MR(-). The algorithm to compute a MR supertree proceeds in the following steps (see methods for details):

1. Generation of the starting tree (the starting tree may also be provided by the user).

2. Supertree computation by minimizing the respective score functions on bifurcating supertrees. A heuristic tree search using the rearrangement operations TDR (taxa-deletion-reinsertion) and NNI (nearest-neighbor interchange) is carried out.

3. Strict consensus tree computation of the best scoring supertrees. The strict consensus contains the splits present in all supertrees.

4. Contracted consensus tree computation by deletion of splits that are contradicted by $\geq 50 \%$ of the input trees.

The resulting tree, i.e., the contracted consensus tree, is the MR supertree of the respective MR method. The last step contracts splits that violate the MR consensus property. Since the MR consensus tree only contains splits occurring in $>50 \%$ of the trees, it cannot contain splits contradicting $\geq 50 \%$ of the input trees. Note that this step would be redundant if the tree search was over multifurcating trees. I will also present results without the last step of the algorithm. The respective supertree methods are denoted $\widetilde{\mathrm{MR}}$. That means, the $\widetilde{\mathrm{MR}}$ supertree is the strict consensus tree. The $\widetilde{M R}$ methods are not generalizations of the majority-rule consensus.

\section{Testing}

Several simulations were conducted to assess the performance of PluMiST. The methods were also compared to MRP using PAUP* [30] with the following options: maximal 1,000,000 trees in memory, 10 replications, and TBR branch swapping.

\section{Simulation with compatible input trees}

This setting is similar to the setting used in [15]. However, I use different model trees generated under a Yule model [31] and subsequently prune a fraction of taxa randomly from ten input trees. If the pruning step deleted the same taxon from each input tree, the data set was discarded and a new data set was generated instead. Thus each taxon had to be present in at least one input tree. I use the following parameter settings: The number of taxa is 32 or 64 , and the fraction of deleted taxa in each input tree is $25 \%$ or $50 \% .100$ replicates are performed for each of the four possible combinations.

An MR method is successful if a score of 0 is found and the resulting strict consensus tree contains only splits present in the true tree. Note that this definition of success differs from the one in [15]. In their case, the method is successful only if the true tree was the only supertree. However, I think that a method should not return only one best scoring tree if some nodes cannot be resolved. Multiple trees with a score of 0 are clearly an indication that some nodes cannot be resolved. In the simulations, there was no check for sufficient overlap between the input trees. Different measures for this criterion exist (e.g., $[32,33]$ ). If there is not sufficient overlap, then the supertree cannot be expected to be reconstructed without ambiguity and this should be reflected by multiple supertrees.

With this criterion of success, all three MR methods and MRP were successful in all cases and under all parameter settings. Furthermore, in each simulation, all three MR methods returned the same number of trees. PAUP* may return more trees since it also returns unresolved trees if they have the same parsimony length. However, in all cases, where PAUP* returned more trees, the strict consensus tree (i.e., the supertree) was the same as for PluMiST. With a deletion probability of 0.25 , more than one optimal tree is found only three and two times with 32 and 64 taxa, respectively. In these cases the resulting supertree had one split missing. With a deletion probability of 0.5 , substantially more optimal trees were found which resulted in more multifurcating trees (Figure 2). On average, trees with $n=32$ contain 24.9 inner splits (instead of 29 for a bifurcating tree) and with $n=64$, there are 53.9 inner splits (instead of 61).

\section{Simulation with incompatible input trees}

I use the same model trees and input trees as in the previous section. However, the input trees were modified such that each internal edge undergoes a nearestneighbor interchange (NNI [34]) with probability $p_{n n i}$ and stays the same with probability $1-p_{n n i}$. The two alternative NNIs are equally likely. The results for $n=$ 32 and $p_{n n i}$ of 0.1 and 0.2 , respectively, are shown in Figure 3.

Since MRP usually performed well in these simulations, its average distance is used as a baseline and I report to what amount the MR methods exceed it. The results for MR(-) are generally comparable to the results 


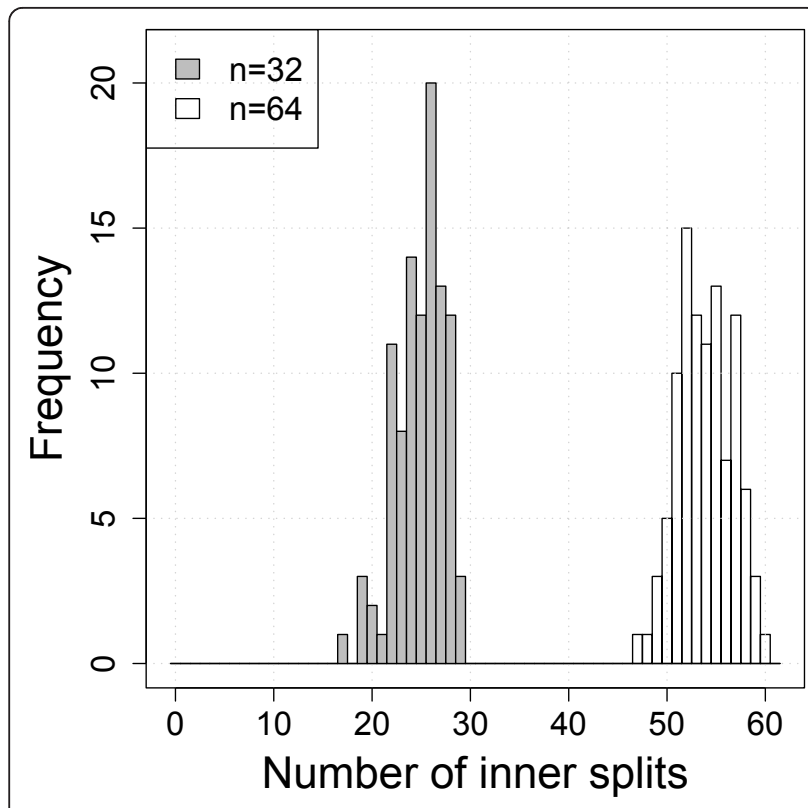

Figure 2 Distribution of inner splits with compatible input trees and $50 \%$ of the taxa deleted.

for MRP. The difference in the mean distances is less than $0.8 \%$ for $\widetilde{M R}(-)$ and less than $0.5 \%$ for $\mathrm{MR}(-)$ and all simulations with $n=32$. In contrast, for the most difficult simulation (Figure 3d), the mean distances for MR $(+) g$ and $\widetilde{M R}(+) g$ exceed the ones for MRP by $1 \%$ and $0.8 \%$, respectively, and the mean distances for $\mathrm{MR}(+)$ and $\widetilde{M R}(+)$ exceed the mean distance for MRP by $3.2 \%$ each. The methods show differences when only the number of missing splits is considered: The average number of missing splits for $\mathrm{MR}(-), \widetilde{\mathrm{MR}}(-), \mathrm{MR}(+) \mathrm{g}$, and $\widetilde{\mathrm{MR}}(+) g$ increases the average for MRP by less then $0.7 \%$ each. In contrast, $\mathrm{MR}(+)$ and $\widetilde{\mathrm{MR}}(+)$ miss up to $1.8 \%$ more of the true splits.

MR(-) rarely finds more incorrect splits than MRP. $\mathrm{MR}(-)$ and $\widetilde{\mathrm{MR}}(-)$ do not find more incorrect splits on average for $p_{n n i}=0.1$, and $0.1 \%$ more for $p_{n n i}=0.2$. In contrast, $\mathrm{MR}(+) \mathrm{g}$ and $\widetilde{\mathrm{MR}}(+) g$ find up to $1.9 \%$ more incorrect splits, while $\mathrm{MR}(+)$ and $\widetilde{\mathrm{MR}}(+)$ find up to $0.8 \%$ more incorrect splits.

\section{Sequence simulation}

PluMiST was also incorporated into a supertree simulation pipeline [35]. Two simulation settings were carried out: a small simulation with 25 taxa, 10 input trees, and on average $37.5 \%$ of the taxa deleted and a large simulation with 69 taxa, 254 input trees, and on average $84.2 \%$ of the taxa deleted. Here I present the results for the simplest setting where the true gene trees are subtrees of the species tree and the simulation parameters are the same for all genes. Input trees were generated
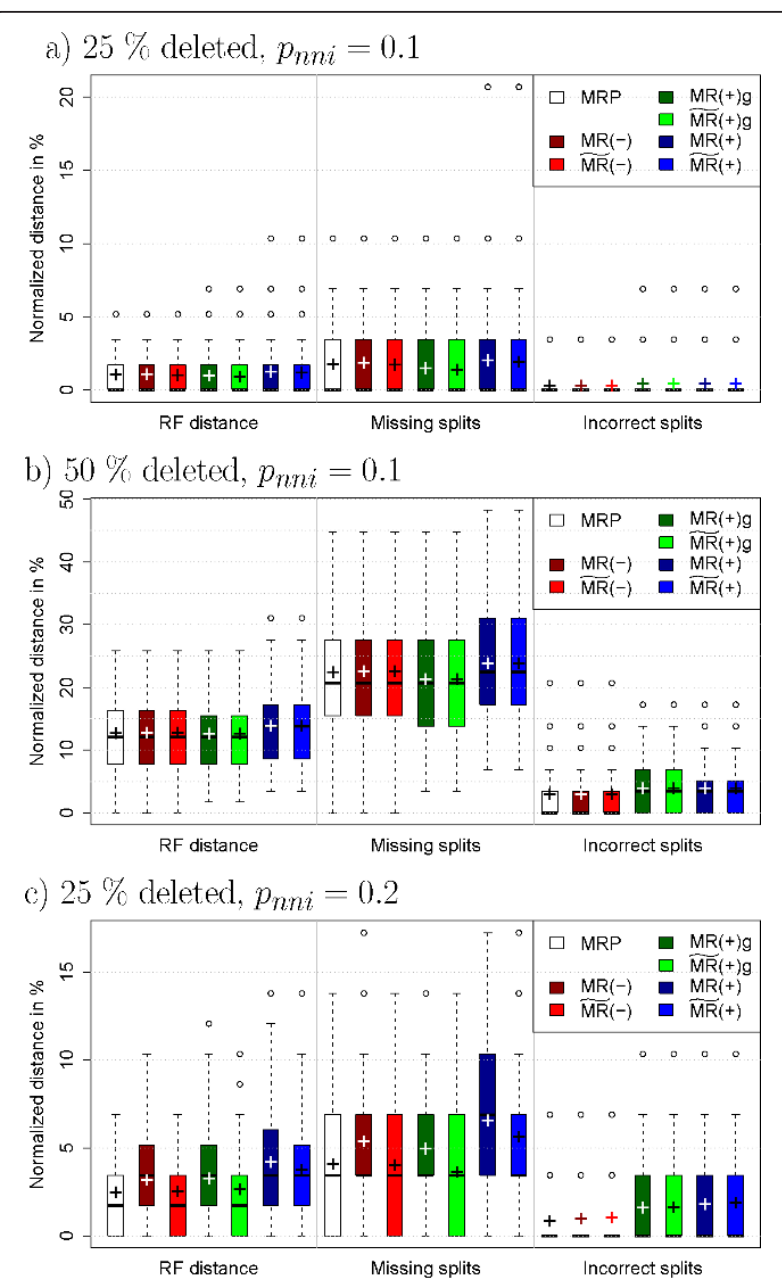

d) $50 \%$ deleted, $p_{n n i}=0.2$

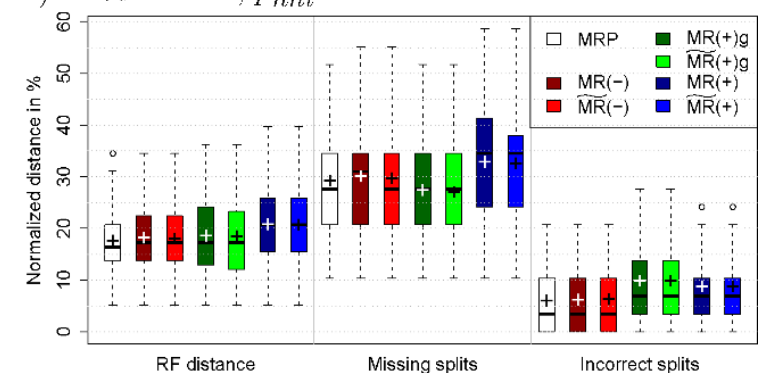

Figure 3 Results for incompatible input trees and $\boldsymbol{n}=\mathbf{3 2}$. The distances are normalized by dividing by the maximal distances, that is, $2 n-6$ for the RF distance and $n-3$ for the other two distances. "Missing splits" is the proportion of splits that are in the model tree but not in the supertree and "Incorrect splits" are in the supertree but not in the model tree. The mean of the data is displayed by " + ". Note the different scaling of the $y$-axes.

by maximum likelihood reconstruction from simulated alignments. 500 simulated data sets were evaluated for the small simulation and 200 for the large simulation.

In contrast to the previous simulations, I conducted ten independent replicates for all MR supertree 
computations and combined trees with the best score over all runs into the final supertree. However, these results were very similar compared to taking one run only (data not shown). Thus, I conclude that the combined search heuristic of TDR and NNI is a sufficient exploration of the tree space in these simulations and report and discuss the results for one run only.

The results for the small simulation (Figure 4a) are similar to the results from the previous section: MR(-) has a slightly higher distance than MRP: $10.9 \%$ compared to $10.8 \%$; $\mathrm{MR}(+) \mathrm{g}$ and $\mathrm{MR}(+)$ have higher distances of $11.5 \%$ and $12 \%$, respectively. The differences between the MR methods are more pronounced in the large simulation (Figure 4b). Here, MR(-) (average distance of $5.1 \%)$ clearly outperforms $\mathrm{MR}(+) \mathrm{g}(10.2 \%)$ and $\mathrm{MR}(+)(15.8 \%)$. In both simulations, the $\widetilde{\mathrm{MR}^{-v e r s i o n s} \text { of }}$ the MR methods resulted in the same trees.

Furthermore, MR(-) clearly improves another MRC implementation (Clann version 3.0.2 [36] with the sfit criterion, SPR search, nsteps $=3$, maxswaps $=$ 1000000, 1 repetition). In the large simulation, MR(-) also outperforms MRP (6.5\%). Both the percentage of missing splits (7.1\% with MR(-) compared to $8.6 \%$ with MRP) and of incorrect splits (3\% compared to $4.3 \%$ ) improves.

There are two main reasons why different methods might reconstruct different trees: the scoring function and the search heuristic. To evaluate whether the difference in the scoring functions can explain the distance differences observed for the large simulation (Figure 4b), the trees of all four methods, MRP, MR(-), MR(+)g, and $\mathrm{MR}(+)$ were scored with the other objective functions. If a method found multiple trees, all trees were scored and the minimum was taken. In $>90 \%$ of the cases the MR (+) supertree had a smaller $\mathrm{MR}(+)$ score than any other supertree, and this also holds for $\mathrm{MR}(+) \mathrm{g}$. MRP and MR (-) supertrees also usually have a smaller parsimony lengths and $\mathrm{MR}(-)$ scores, respectively, than the $\mathrm{MR}(+) \mathrm{g}$ and $\mathrm{MR}(+)$ trees (> $99 \%$ of the cases). In $31 \%$ of the cases the parsimony lengths of the MRP and MR(-) supertree were equal and in $31.5 \%$ the MR(-) scores of both methods were equal. It was never observed that another method found a lower parsimony or MR(-) score than the respective supertree methods. However, for $\mathrm{MR}(+)$, at least one method resulted in a better MR (+) score in $6.5 \%$ of the cases. In $0.5 \%$ of the cases, one $\mathrm{MR}(+)$ supertree had a better $\mathrm{MR}(+) \mathrm{g}$ score than the $\mathrm{MR}(+) \mathrm{g}$ supertree.

To summarize, supertrees from different methods usually vary in their scores when evaluated with one objective function (MRP, MR(-), MR(+)g, or MR(+)). The search heuristics implemented in PAUP* and PluMiST usually find better scores for the respective objective functions compared to the supertrees found with the other methods.

\section{Evaluation of real data sets}

The program was also applied to real data sets and compared with MRP using PAUP*. First, two data sets also used in Bansal et al. [29] were analyzed (available from [37]). The seabirds data set [38] contains rooted trees, thus an outgroup taxon was added to the trees. The mammals data set contains "semi-rooted" trees [39], i.e., not all trees are rooted and an outgroup taxon is already present in some of the input trees. Some trees were discarded from the mammal data set since they contain no inner splits and thus no information for MR supertrees or MRP. Since both data sets contain some multifurcating input trees, only MR(-) was evalutated.

The results are summarized in Table 2. The high number of optimal trees for the seabirds data set had already been reported [38]. This slows down the last part of the PluMiST algorithm, because equally scoring trees are explored by NNI. The RF supertree method [29] finds a score of 61, but only 4 trees of the optimal score for this data set. This score also corresponds to the optimal MR(-) score of 23, but PluMisT finds 1538 optimal trees. The runtime of RF supertrees is substantially lower than the runtime of PluMiST or PAUP* for two reasons. First, efficient heuristics use the root information, and second, the search does not continue to find multiple optimal trees.

The mammal data set has different optima depending on whether the MRP or the MR(-) criterion is used (Table 2). The full data set could not be analyzed with a) Small simulation

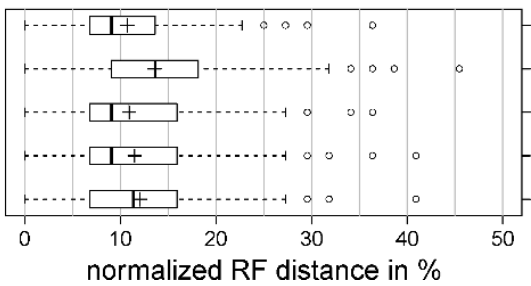

b) Large simulation

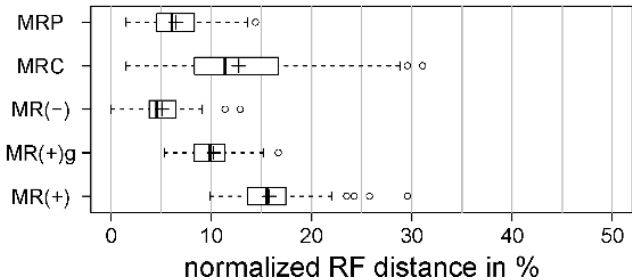

Figure 4 Results with sequence simulation. The mean of the data is displayed by "+". 
Table 2 Results with the seabird and the mammal data set

\begin{tabular}{lcccccc}
\hline Data Set & Taxa & Input trees & Mest score & Optimal trees & MRP-score & Time \\
\hline Seabirds [38] & 122 & 7 & 23 & 1538 & 214 & 1 day* \\
Mammals [39] & 116 & 692 & 2160 & 272 & 9454 & $13 \mathrm{~h} 10$ \\
\hline
\end{tabular}

\begin{tabular}{lcccccc} 
& & & & & & \\
MRP & & & \\
Data Set & Taxa & Input trees & Best score & Optimal trees & MR(-)-score & Time \\
\hline Seabirds [38] & 122 & 7 & 214 & $10^{6}$ & 23 & $9 \mathrm{h00}$ \\
Mammals [39] & 116 & 692 & 9452 & 109 & 2162 & $1 \mathrm{~h} 40$ \\
\hline
\end{tabular}

* The search with the seabirds data set was aborted after about 1 day, and the optimal trees saved thus far were used. The optimal score of 23 was found after $1 \mathrm{~h} 20$.

RF supertrees since not all trees are rooted. Lastly, a microbial data set containing 61 taxa and 1117 genes was analyzed [40]. Bootstrap resampling of the gene trees and computing MRC using Clann had resulted in a highly multifurcating majority-rule consensus tree [40]. The data set consists of bifurcating unrooted trees and I applied it to the three majority-rule supertree methods and to MRP. All supertrees are completely or nearly completely resolved (Figure 5). There are clear differences in the scores of the optimal trees when evaluated with other scoring functions (Table 3). None of the trees matches a recent reference phylogeny completely (Figure 5e, Figure 1 in [41]). All of the nine groupings marked by different colors in Figure 5 are present in the MR(-) and in the MRP tree. Both of the MR(+) variants nest the Betaproteobacteria inside the Gammaproteobacteria. The branching order of these groupings also shows some deviations from the reference tree. First, most of the groupings with only one member in the data set are not correctly placed in any tree: Aquifex aeolicus is never a sister of the Epsilonproteobacteria, Chlorobium tepidum is never a sister of the Chlamydiae, the Cyanobacterium Synechocystis is never a sister of the Actinobacteria, and Deinococcus radiodurans is never basal. Because of these problems, the information about groupings with one member only are ignored in the following points. Second, the Alpha-, Beta- and Gammaproteobacteria form a clade in all trees, but the Epsilonproteobacteria are not their sister group. In the $\mathrm{MR}(-)$ and $\mathrm{MR}(+)$ tree, the Epsilonproteobacteria form a clade with the Chlamydiae and in the $\mathrm{MR}(+) \mathrm{g}$ tree, they form a clade with the Chlamydiae and the Tenericutes. In the MRP tree, they are basal to a clade of Chlamydiae and the other Proteobacteria. Third, in both the MR(-) and the MRP tree, the Firmicutes do not cluster with the Actinobacteria but with the Tenericutes. Only in the MRP tree, the clade of Tenericutes, Firmicutes and Actinobacteria is basel to the other Bacteria. In the MR(-) tree, the clade of Epsilonproteobacteria and Chlamydiae is basal and in the $\mathrm{MR}(+) \mathrm{g}$ tree a clade of these two and the Tenericutes is basal. The $\mathrm{MR}(+)$ tree has more differences; even the Archaea are not monophyletic.

Taken together there are serious problems in the data set, when groups have only one member. The fewest contradictions to the reference tree are present in the MRP tree followed by the MR(-) tree. Note that the deep branching of the bacterial phylogeny and the meaningfulness of a search for a tree like pattern in Bacteria is hotly debated (e.g., [42-45]). Here, the microbial data set is presented as an example how different methods resolve conflict among the input trees and not as a statement about the "true" bacterial phylogeny.

\section{Conclusions}

I present a new framework for the computation of the distances underlying majority-rule supertrees. The basis of this framework is the relationship matrix that stores the possible relationships between an input tree split and a supertree split: subsplit, compatibility, or incompatibility. The distance computations for $\operatorname{MR}(-), \operatorname{MR}(+)$ $\mathrm{g}$, and $\mathrm{MR}(+)$ are presented for bifurcating input- and supertrees. The distance computation of $\mathrm{MR}(-)$ also applies to multifurcating input trees.

These distance computations are implemented in the python program PluMiST. The program was tested in a simulation study with different settings, in particular with compatible and incompatible input trees. With compatible input trees, all MR methods find a score of 0 and the same supertree as MRP and I thus conclude that all methods search the tree space successfully. With incompatible input trees, the results for $\mathrm{MR}(-)$ are best, especially when both missing and incorrect splits are taken into account. $\mathrm{MR}(+) \mathrm{g}$ might miss less splits than MR(-), however it also finds more incorrect splits. Reconstructing incorrect splits which give rise to false conclusions is obviously a more serious problem compared to the exclusion of some splits.

The RF distance to the true tree is often decreased by including splits contradicted by a majority of the input 


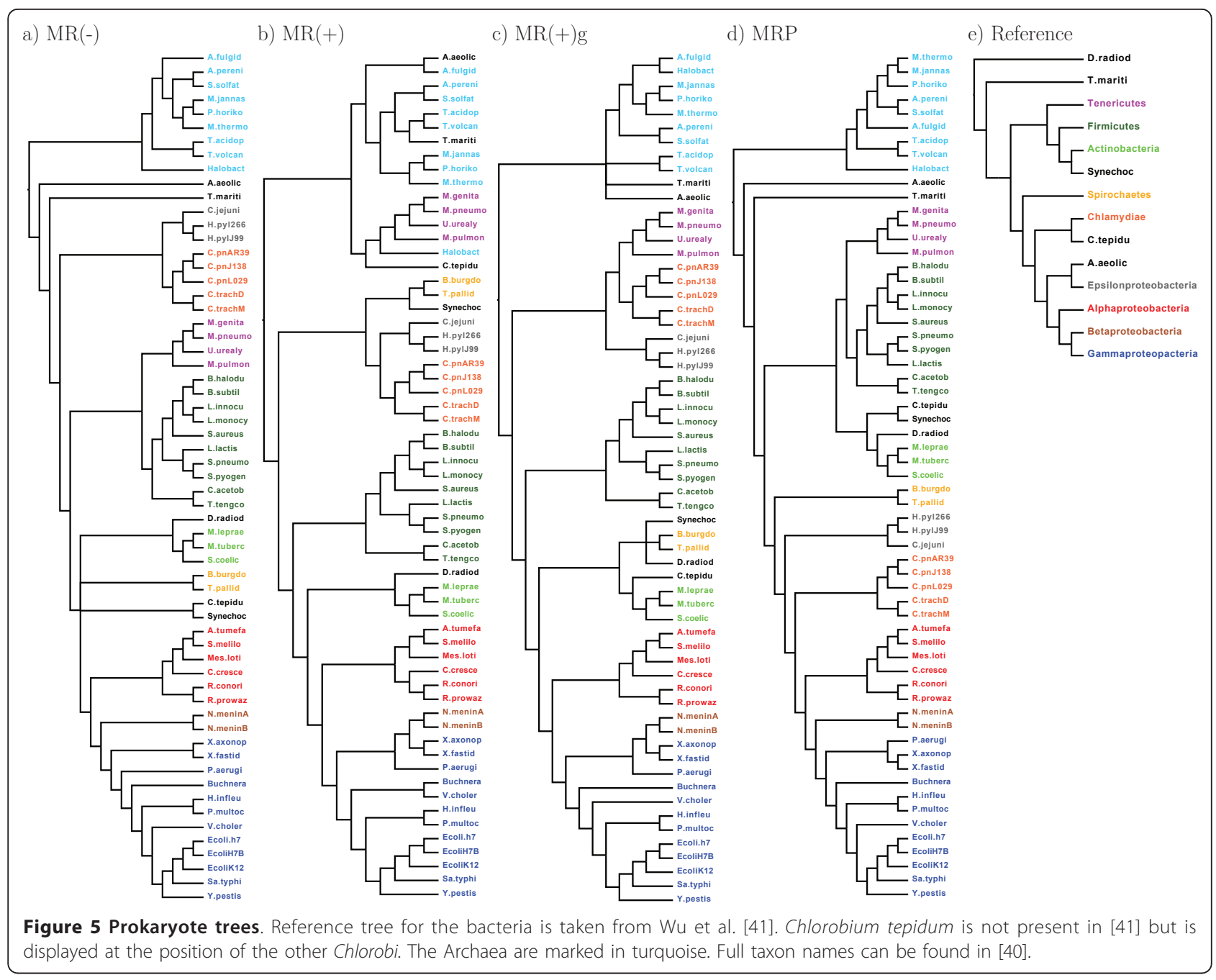

trees (i.e., skipping the last step of the algorithm, denoted by $\widetilde{\mathrm{MR}}$ ). This holds particularly when only a few taxa are deleted and the input trees are highly incongruent (25\% of the taxa deleted and $p_{n n i}=0.2$ ). While the $\widetilde{\mathrm{MR}}$ methods are not a generalization of MR consensus anymore, they may still be useful supertree methods. Given that $\widetilde{\mathrm{MR}}(-$ ) is equivalent to MRC for bifurcating supertrees, PluMiST can then also be used as a heuristic for MRC.

In a more realistic simulation setting involving more input trees, trees of different sizes and different amounts

Table 3 Results with the microbial data set

\begin{tabular}{lcccccc}
\hline & \multicolumn{5}{c}{ Scores } & \\
\cline { 2 - 6 } Method & MR(-) & MR(+) & MR(+)g & Parsimony & Optimal trees & Time \\
\hline MR(-) & $\mathbf{1 4 5 3}$ & 1875 & 3328 & 6802 & 4 & 19 min \\
MR(+) & 1539 & $\mathbf{1 7 4 4}$ & 3283 & 7003 & 1 & $24 \mathrm{~min}$ \\
MR(+)g & 1484 & 1753 & $\mathbf{3 2 4 3}$ & 6862 & 4 & $36 \mathrm{~min}$ \\
MRP & 1474 & 2010 & 3484 & $\mathbf{6 7 7 5}$ & 1 & $2 \mathrm{sec}$ \\
\hline
\end{tabular}

Scores matching the criterion used for optimization are marked in bold. of missing data and sequence simulation, $\mathrm{MR}(-)$ performed very well. It outperformed the MRC implementation in Clann and also MRP for the large data set. Those differences can be traced back to the differences in the objective functions in PAUP* and PluMisT. The trees found with the respective objective function usually have better scores compared to the supertrees found with other methods.

This is also observed when analyzing biological data sets. Of the three data sets analyzed, two contain multifurcating input trees, and therefore only $\mathrm{MR}(-)$ was compared to MRP and to RF supertrees. For one data set with rooted input trees the score of MR(-) was equivalent to the score to RF supertrees, but MR(-) finds more optimal trees. The microbial data set contains bifurcating trees and was analyzed with all three majority-rule supertree methods. The $\mathrm{MR}(-)$ tree recovered more relationships present in the reference tree compared to the $\mathrm{MR}(+)$ variants.

In conclusion, the objective function of MR(-) performs best among the MR supertree methods studied 
here. Note that this objective function is also justified in the likelihood setting [46]. The MR(+) supertree methods add taxa to the input trees and apply a consensus. At first view, this approach seems to be a natural method for dealing with the supertree problem. However, phylogenetic signal may be confounded by properties of the complex tree space [47].

The problem of distance computations in the general case of multifurcating trees is still open but I suppose that formulas for these cases can be constructed based on the relationship matrix. The generalization of these computations to multifurcating supertrees is an important task. The proof for majority-rule consensus trees [19] only holds for multifurcating consensus trees and was the motivation for majority-rule supertrees [26]. Note that another variant of MR(+), MR(+)s supertrees, can be computed via the span and the consensus step and thus also includes multifurcating trees [28].

The current implementation of MR(-) in PluMiST is similar to the RF supertree method. The approach of Bansal et al. [29] is, however, to find at least one tree of the optimal score and not to search for equal scoring trees. This latter property is needed for MR supertrees. This, in addition to the heuristics for rooted trees, makes the RF supertree method fast. In contrast, the advantage of MR(-) is that input trees can be unrooted and that the area around the optimum is searched for equally scoring trees.

Furthermore, PluMiST and the theory presented here comprises a general framework for MR supertrees. The relationships between the distance functions underlying these methods will hopefully help in understanding the similarities and differences of the methods. Simulations show that MR(-) usually performs very well, in particular in comparison with the established MRP method and is thus recommended for majority-rule supertree reconstruction.

\section{Methods}

\section{Phylogenetic Background}

A (phylogenetic) tree is a leaf-labeled tree and is thus identified by its leaf set $X$ and its edge set (for details and terminology see also [48]). The leaves are usually called taxa. Terminal edges connect a leaf with an inner node and inner edges connect two inner nodes. I present the computation for unrooted trees. This computation can easily be applied to rooted trees by treating the root as an additional taxon. In unrooted trees there is no node of degree two.

If an edge of a phylogenetic tree is deleted, the tree decomposes into two connected components. Thus, the taxon set is then partitioned into two sets $\left(X_{1}\right.$ and $\left.X_{2}\right)$, one for each component. Such a bipartition is called a split and is denoted by $X_{1} \mid X_{2}$. Since each edge in a tree corresponds to a split, a tree on taxon set $X$ is identified by the corresponding split set (see example in Figure 1, note that the taxon sets in a split can be shortly written as a string of concatenated taxa).

Two splits are called compatible if there is a phylogenetic tree containing both splits. This holds for two splits $X_{1} \mid X_{2}$ and $Y_{1} \mid Y_{2}$ if at least one of the following taxon sets is empty: $X_{1} \cap Y_{1}, X_{1} \cap Y_{2}, X_{2} \cap Y_{1}$ or $X_{2} \cap Y_{2}$. Note that terminal splits are compatible to any other split. An unrooted phylogenetic tree of $n$ taxa contains at most $n-3$ inner splits. If it contains exactly $n-3$ inner splits, all inner nodes have degree three, and the tree is called bifurcating, or multifurcating otherwise. An inner node of degree three is a taxon tripartition and can be written as $X_{1}\left|X_{2}\right| X_{3}$.

A tree $T_{1}$ displays a tree $T_{2}$ if it contains all splits of $T_{2}$, i.e., $T_{2} \subseteq T_{1}$. The Robinson-Foulds (RF) distance of two trees is defined as the symmetric difference of the split sets [20]: $\operatorname{RF}\left(T_{1}, T_{2}\right)=\left|T_{1} \backslash T_{2}\right|+\left|T_{2} \backslash T_{1}\right|$. Note that if both trees are bifurcating, then both set sizes are equal and RF is an even number.

A supertree $\mathcal{S}$ for a set of input trees $\mathcal{P}$ is a tree that contains exactly the taxa occurring in at least one input tree, i.e. $X_{\mathcal{S}}=\bigcup_{\mathcal{G} \in \mathcal{P}} X_{\mathcal{G}}$. Thus in general $X_{\mathcal{G}} \subseteq X_{\mathcal{S}}$. The following abbreviations are used: $n=\left|X_{\mathcal{S}}\right|$ and $n_{\mathcal{G}}=\left|X_{\mathcal{G}}\right|$. The splits in $\mathcal{G}$ are called partial if $X_{\mathcal{G}} \subset X_{\mathcal{S}}$. In contrast, the splits in $\mathcal{S}$ are called plenary. A partial split $g \in \mathcal{G}\left(g=Y_{1} \mid Y_{2}\right)$ is a subsplit of a plenary split $s \in \mathcal{S}\left(s=Z_{1} \mid Z_{2}\right)$ if one of the following conditions holds: $\left(Y_{1} \subseteq Z_{1}\right.$ and $\left.Y_{2} \subseteq Z_{2}\right)$ or $\left(Y_{1} \subseteq Z_{2}\right.$ and $\left.Y_{2} \subseteq Z_{1}\right)$. For example, the split $A B C \mid F$ is a subsplit of the split $A B C \mid D E F$. Two splits on different taxon sets will be called compatible if they are compatible on the set of taxa occurring in both trees and incompatible otherwise.

$\mathcal{S} \mid X_{\mathcal{G}}$ is the restriction of tree $\mathcal{S}$ to taxon set $X_{\mathcal{G}}$, i.e., $\mathcal{S} \mid X_{\mathcal{G}}=\left\{A \cap X_{\mathcal{G}}\left|B \cap X_{\mathcal{G}}: A\right| B \in \mathcal{S}\right.$ and $\left.A \cap X_{\mathcal{G}} \neq \emptyset, B \cap X_{\mathcal{G}} \neq \emptyset\right\}$.

\section{Consensus methods}

Consensus methods combine input trees on the same taxon set. There are many consensus methods available [17], some of which are based on the split sets of the input trees:

Strict consensus The strict consensus contains all splits present in all input trees, i.e., $T=\bigcap_{\mathcal{G} \in \mathcal{P}} \mathcal{G}$.

Majority-rule consensus The majority-rule (MR) consensus contains all splits present in more than half of the input trees.

\section{Distance computations for MR supertrees}

The majority-rule supertree methods were defined to minimize a particular score [26,27]:

$\operatorname{MR}(-)$ Find a tree $\mathcal{S}$ that minimizes $\sum_{\mathcal{G} \in \mathcal{P}} d^{-}(\mathcal{S}, \mathcal{G})$ where $d^{-}(\mathcal{S}, \mathcal{G})=\operatorname{RF}\left(\mathcal{S} \mid X_{\mathcal{G}}, \mathcal{G}\right)$. 
$\mathbf{M R}(+)$ g Find a tree $\mathcal{S}$ that minimizes $\sum_{\mathcal{G} \in \mathcal{P}} d_{g}^{+}(\mathcal{S}, \mathcal{G})$ where $\quad d_{g}^{+}(\mathcal{S}, \mathcal{G})=\min _{\left.\mathcal{G}^{\prime} \in \mathcal{G}\right\rangle_{g}} \operatorname{RF}\left(\mathcal{S}, \mathcal{G}^{\prime}\right) \quad$ and $\langle\mathcal{G}\rangle_{g}=\left\{\mathcal{G}^{\prime}: X_{\mathcal{G}^{\prime}}=X_{\mathcal{S}}\right.$ and $\left.\mathcal{G}^{\prime} \mid X_{\mathcal{G}}=\mathcal{G}\right\}$

$\operatorname{MR}(+)$ Find a tree $\mathcal{S}$ that minimizes $\sum_{\mathcal{G} \in \mathcal{P}} d^{+}(\mathcal{S}, \mathcal{G})$ where $d^{+}(\mathcal{S}, \mathcal{G})=\min _{\mathcal{G}^{\prime} \in\langle\mathcal{G}\rangle} \operatorname{RF}\left(\mathcal{S}, \mathcal{G}^{\prime}\right)$

and

$\langle\mathcal{G}\rangle=\left\{\mathcal{G}^{\prime}: X_{\mathcal{G}^{\prime}}=X_{\mathcal{S}}\right.$ and $\mathcal{G}^{\prime}$ is bifurcating and $\mathcal{G}^{\prime} \mid X_{\mathcal{G}}$ displays $\left.\mathcal{G}\right\}$

As for majority-rule consensus trees, the majority-rule supertree is the strict consensus of all potentially multifurcating trees that minimize the distance. Here, the tree search is restricted to bifurcating trees. In the following I show, that for bifurcating input- and supertrees the distance computations can be simplified. Therefore assume that $\mathcal{S}$ is already modified as follows: If there is an inner split $X_{1} \mid X_{2} \in \mathcal{S}$ with $X_{i} \subseteq X_{\mathcal{S}} \backslash X_{\mathcal{G}}$ and there is no split $Y_{1} \mid Y_{2} \in \mathcal{S}$ with $X_{i} \subset Y_{j} \subseteq X_{\mathcal{S}} \backslash X_{\mathcal{G}}$, then replace the subtree spanned by the the taxa in $X_{i}$ by a dummy taxon $(i, j \in\{1,2\})$. For $d^{-}$this modification will make no difference, since the dummy taxon is deleted again. For $d^{+}$and $d_{g}^{+}$only this dummy taxon needs to be placed in $\mathcal{G}$. Afterwards it could be expanded again without any cost. This modification ensures that in the optimal $\mathcal{G}^{\prime} \in\langle\mathcal{G}\rangle$ each taxon was added to an edge already existing in $\mathcal{G}$ (analogous for $\langle\mathcal{G}\rangle_{g}$ ). The modified example tree is shown in Figure 6.

A matrix representation is a binary coding of the splits, an example is shown in Table 4. Furthermore, the relationship matrix of dimension $\left(2 n_{\mathcal{G}}-3\right) \times\left(n+n_{\mathcal{G}}-3\right)$ is used, which indicates for each pair of input-and supertree splits, whether they are subsplit; compatible but no subsplit; or incompatible. Only inner splits and trivial splits for $X_{\mathcal{G}}$ are included (see Table 4). Note that for bifurcating trees there cannot be a split compatible to all other splits in the relationship matrix. Thus each line and row, respectively, must contain either at least one $s$ or at least one $i$.

$M R(-)$

Theorem 1 Given a bifurcating supertree $\mathcal{S}$ and a bifurcating input tree $\mathcal{G}$. Then $d^{-}=2 C$, where $C$ is the number of splits in $\mathcal{G}$ that are incompatible to at least one split in $\mathcal{S}$.

Proof Let $\mathcal{S}^{\prime}$ be the restriction of $\mathcal{S}$ to $X_{\mathcal{G}}$, i. e. $\mathcal{S}^{\prime}=\mathcal{S} \mid X_{\mathcal{G}}$.

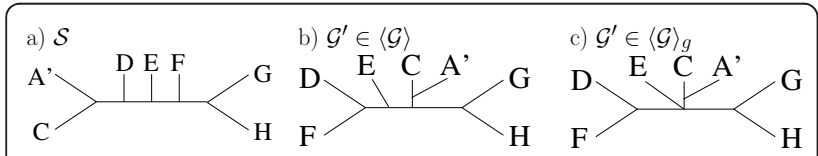

Figure 6 Modification of the supertree. The taxon set $\{A, B\}$ is replaced by $A^{\prime}$.
$d^{-}(\mathcal{S}, \mathcal{G})=\operatorname{RF}\left(\mathcal{G}, \mathcal{S}^{\prime}\right)=\left|\mathcal{G} \backslash \mathcal{S}^{\prime}\right|+\left|\mathcal{S}^{\prime} \backslash \mathcal{G}\right|=2 \times\left|\mathcal{G} \backslash \mathcal{S}^{\prime}\right|$.

Since both trees are bifurcating, each $g \in \mathcal{G}$ must be either identical or incompatible to a split in $\mathcal{S}^{\prime}$. Thus $\left|\mathcal{G} \backslash \mathcal{S}^{\prime}\right|$ measures the number of splits in $\mathcal{G}$ that are incompatible to a split in $\mathcal{S}^{\prime}$. Each $g \in \mathcal{G}$ that is incompatible to a split in $\mathcal{S}^{\prime}$, is also incompatible to a split in $\mathcal{S}$, since incompatibility can only be caused by the taxa in $X_{\mathcal{G}}$. Thus $\left|\mathcal{G} \backslash \mathcal{S}^{\prime}\right|$ is the number of splits in $\mathcal{G}$ that are incompatible to a split in $\mathcal{S}$.

Note The formula easily generalizes to multifurcating input trees: Assume that $\mathcal{G}$ has $m$ multifurcations, i.e., $n_{\mathcal{G}}-m-3$ inner branches. Then $d^{-}=2 C+m$, there are $C$ splits in $\mathcal{G}$ that conflict with $\mathcal{S}^{\prime}, C$ splits in $\mathcal{S}^{\prime}$ that conflict with $\mathcal{G}$, and in addition $m$ splits in $\mathcal{S}^{\prime}$ that are missing in $\mathcal{G}$. Since $m$ is constant for all supertrees, the objective function is equivalent to the one for bifurcating input trees.

Note, that $C$ can be easily computed from the relationship matrix since $C=\sum_{i}^{2 n_{\mathcal{G}}-3} c_{i}$.

\section{$M R(+) g$}

Theorem 2 Given a bifurcating supertree $\mathcal{S}$ and a bifurcating input tree $\mathcal{G}$. Then $d_{g}^{+}=B+C$, where $B$ is the number of splits in $\mathcal{S}$ that are incompatible to at least one split in $\mathcal{G}$ and $C$ is the number of splits in $\mathcal{G}$ that are incompatible to at least one split in $\mathcal{S}$.

Proof Both inequalities are shown. The idea of the proof is to show that $C=\left|\mathcal{G}^{\prime} \backslash \mathcal{S}\right|$ and $B=\left|\mathcal{S} \backslash \mathcal{G}^{\prime}\right|$ for one $\mathcal{G}^{\prime} \in\langle\mathcal{G}\rangle_{g}$. Since $B \geq C,\left|\mathcal{S} \backslash \mathcal{G}^{\prime}\right| \geq\left|\mathcal{G}^{\prime} \backslash \mathcal{S}\right|$, this is accomplished by potentially introducing multifurcations when placing taxa onto $\mathcal{G}$.

$B+C \leq d_{g}^{+} \quad$ For $\quad$ all $\quad \mathcal{G}^{\prime} \in\langle\mathcal{G}\rangle_{g}: C \leq\left|\mathcal{G}^{\prime} \backslash \mathcal{S}\right|$ and $B \leq\left|\mathcal{S} \backslash \mathcal{G}^{\prime}\right|$. Thus this inequality holds for all $\mathcal{G}^{\prime}$.

$d_{g}^{+} \leq B+C$ Need to construct a $\mathcal{G}^{\prime} \in\langle\mathcal{G}\rangle_{g}$ with $\left|\mathcal{S} \backslash \mathcal{G}^{\prime}\right|+\left|\mathcal{G}^{\prime} \backslash \mathcal{S}\right| \leq B+C$.

First, $\mathcal{S}^{\prime}=\mathcal{S} \mid X_{\mathcal{G}}, \operatorname{RF}\left(\mathcal{S}^{\prime}, \mathcal{G}\right)=2 C$. It is shown now that the taxa in $X_{\mathcal{S}} \backslash X_{\mathcal{G}}$ can be placed onto $\mathcal{S}^{\prime}$ and $\mathcal{G}$ and thus increase the distance by not more than $B-C$. Therefore, $d_{g}^{+} \leq 2 C+B-C=B+C . n_{T}=n-n_{G}$ taxa have to be placed onto $\mathcal{G}$ to get $\mathcal{G}^{\prime}$. There will be $n_{\text {good }}$ "good" taxa that are placed onto edges without introducing an increase in the distance; and $n_{\text {bad }}$ "bad" taxa that are placed onto nodes and will increase the distance by one; $n_{T}=n_{\text {good }}+n_{\text {bad }}$.

Each supertree split can only be a supersplit of at most one input split. If it is a supersplit of an input split, it is compatible to all others. An input split may be a subsplit of different supertree splits. If it is a subsplit of $>1$ supertree split, each additional supertree split gives information about the placement of one taxon. e.g., the input split $A \mid B$ may be a subsplit of the supertree splits $A X \mid B$ and $A \mid X B$ ( $A$ and $B$ are taxon sets, $X$ is a taxon). Then $X$ is a "good" taxon and placed on the edge between $A$ and $B$ without 


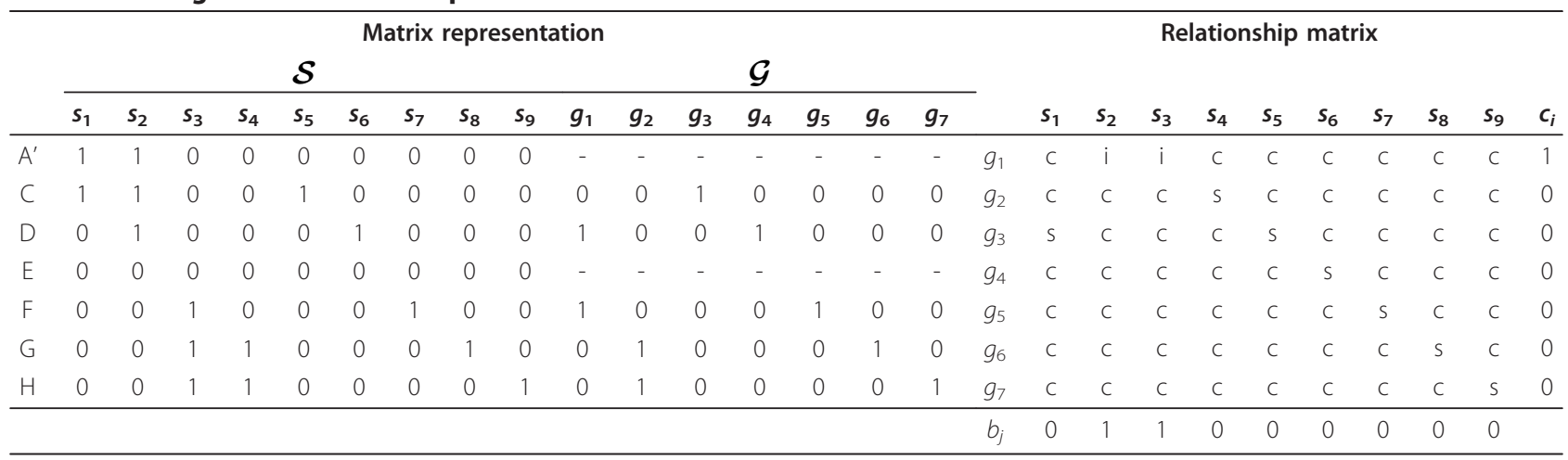

increasing $d_{g}^{+}$. The number of taxa that are placed with this procedure is $\bar{B}-\bar{C} \cdot \bar{B}$ and $\bar{C}$ represent the respective number of columns and rows in the relationship matrix that have a subsplit entry.

In each row and column of the matrix, there must be either at least one subsplit entry (s) or at least one incompatibility entry (i). Thus $\bar{C}+C=2 n_{\mathcal{G}}-3$ and $\bar{B}+B=n+n_{\mathcal{G}}-3$. Using this, the remaining number of taxa to insert is

$$
n_{T}-(\bar{B}-\bar{C})=n-n_{\mathcal{G}}-\left(n+n_{\mathcal{G}}-3-B\right)+2 n_{\mathcal{G}}-3-C=B-C .
$$

These $B$ - $C$ bad taxa can be placed on a node increasing $d_{g}^{+}$by one. In detail, the two splits in $S$ that are adjacent to the terminal split of the bad taxon will be incompatible with at least one split in $\mathcal{G}$. As a result the bad taxon can be placed on any of the nodes adjacent to a conflicting split in $\mathcal{G}$. The resulting distance is not larger than $2 C+(B-C)=B+C$.

Example In the example $\bar{C}=6, \bar{B}=7, C=1$ and $B=$ 2. Thus, $n_{\text {good }}=\bar{B}-\bar{C}=1\left(\right.$ taxon $\left.A^{\prime}\right)$ and $n_{\text {bad }}=B-C=$ 1 (taxon $E$ ). $g_{3}$ is a subsplit of both $s_{1}$ and $s_{5}$, thus $A^{\prime}$ can be placed on the terminal edge leading to $C$ without conflict. $s_{2}$ and $s_{3}$ are adjacent to the terminal split of $E$. They are conflicting with $g_{1}$ and thus $E$ can be placed either on the node $D|F| C G H$ or on the node $C|D F| G H$.

$$
M R(+)
$$

Theorem 3 Given a bifurcating supertree $\mathcal{S}$ and a bifurcating input tree $\mathcal{G}$. Then $d^{+}=2 B$, where $B$ is the number of splits in $\mathcal{S}$ that are incompatible to at least one split in $\mathcal{G}$.

Proof The proof is a modification of the proof for Theorem 2. The "good" taxa are placed in the same way and the bad taxa are placed onto any split corresponding to a conflicting split in $\mathcal{G}$. The resulting distance is then $2 C+2(B-C)=2 B$.

\section{Heuristic algorithm Starting tree}

The tree search starts with a step-wise addition tree. A random taxa order is processed the following way: The quartet toplogy for the first four taxa is determined by the topolgy most frequent among the input trees. The remaining $n-4$ taxa are inserted step by step to the partially reconstructed tree. For each of the remaining taxa, the informative input trees are determined. These trees contain the considered taxon and at least 3 of the taxa already inserted. Afterwards, the best insertion point of the terminal branch labeled with the taxon is determined: If the objective function is $d^{-}$, then the sum of $d^{-}$is computed for each insertion point. This is done by pruning the supertrees and the input trees to the common taxon sets. If the objective function is $d^{+}$, for each split in the supertree the number of input trees it contradicts is determined. The insertion point minimizes the sum of the split contradictions. This method does not ensure $d^{+}$since taxa are missing in both trees. If the objective function is $d_{g}^{+}$, both types of insertion strategies are carried out alternatingly, i.e., for each insertion only one of the strategies is used. In all cases ties are resolved randomly.

\section{Optimization step}

The tree search allows for two rearrangement operations: Taxa-deletion-reinsertion (TDR) and nearestneighbor interchange (NNI). Given a tree, TDR deletes a given fraction of the taxa randomly (by default 0.25 ). Afterwards, a random taxon order of the deleted taxa is determined and the taxa are reinserted via the step-wise addition strategy. An NNI operation takes a split of a tree and generates the two alternative splits that could replace it. If replacing the split with one of these splits would result in a supertree with a lower score, the split is replaced. Alternative best trees are also returned.

The tree search proceeds in two stages: The first exploration stage lasts at most $l^{2}$ iterations, where $l$ is the number of inner splits in a supertree and an iteration is an NNI or a TDR operation. First, all splits of the starting tree are optimized by NNI until there is no further improvement. Next, the tree space is explored by TDR. After one TDR operation, all splits of the new tree are optimized by NNI again. It may happen that a 
TDR operation generates a tree that it found before or that yield no improvement with NNI. Then this tree is discarded and a new tree is generated with TDR. If $\sqrt{l}$ trees were discarded consecutively or $l^{2}$ iterations passed, the broadening stage starts. At this point, all optimal trees that have not been analyzed before, are optimized by NNI. This mainly ensures that all trees in the optimal region are found.

In the end, all optimal trees are summarized by a strict consensus and splits that are contradicted by $\geq$ $50 \%$ of the input trees are deleted. In these trees, the internal nodes are labeled with support values similar to those of majority-rule consensus trees. Each inner node is labeled with $x / y$, where $x$ is the number of input trees not contradicting the corresponding split and $y$ is the number of input trees where a nontrivial split supports the corresponding split. Thus, $y$ is the number of input trees that support the node and $x-y$ is the number of input trees that are irrelevant for that node with the definitions of support and irrelevance used in [49].

\section{Acknowledgements}

The author likes to thank Jonathan P. Bollback for assistance in analyzing the prokaryote supertrees, Arndt von Haeseler and Bui Quang Minh for valuable comments on the manuscript and Mark Wilkinson for motivating discussions. Financial support from the Hungarian Bioinformatics project (HuBi MTKD-CT-2006-042794) and from the the Wiener Wissenschafts-, Forschungs- and Technologiefonds (WWTF, to Arndt von Haeseler) is greatly appreciated.

\section{Author details}

${ }^{1}$ Center for Integrative Bioinformatics Vienna, Max F. Perutz Laboratories, University of Vienna, Medical University of Vienna, University of Veterinary Medicine Vienna, Dr. Bohr-Gasse 9, A-1030 Vienna, Austria. ${ }^{2}$ Institute of Science and Technology, Austria, Am Campus 1, 3400 Klosterneuburg, Austria.

\section{Authors' contributions}

AK conceived and implemented the method, carried out the analyses, and wrote the manuscript.

Received: 1 September 2010 Accepted: 13 July 2011

Published: 13 July 2011

\section{References}

1. Bininda-Emonds ORP, (Ed): Phylogenetic Supertrees: Combining Information to Reveal the Tree of Life Dordrecht: Kluwer Academic; 2004.

2. Fitzpatrick D, Logue M, Stajich J, Butler G: A fungal phylogeny based on 42 complete genomes derived from supertree and combined gene analysis. BMC Evol Biol 2006, 6:99.

3. Pisani D, Cotton JA, Mclnerney JO: Supertrees disentangle the chimerical origin of eukaryotic genomes. Mol Biol Evol 2007, 24(8):1752-1760.

4. Bininda-Emonds ORP, Cardillo M, Jones KE, MacPhee RDE, Beck RMD, Grenyer R, Price SA, Vos RA, Gittleman JL, Purvis A: The delayed rise of present-day mammals. Nature 2007, 446:507-512.

5. Baker WJ, Savolainen V, Asmussen-Lange CB, Chase MW, Dransfield J, Forest F, Harley MM, Uhl NW, Wilkinson M: Complete Generic-Level Phylogenetic Analyses of Palms (Arecaceae) with Comparisons of Supertree and Supermatrix Approaches. Syst Biol 2009, 58(2):240-256.

6. Davis RB, Baldauf SL, Mayhew PJ: The origins of species richness in the Hymenoptera: insights from a family-level supertree. BMC Evol Biol 2010, 10:109.
7. Baum BR: Combining Trees as a Way of Combining Data Sets for Phylogenetic Inference, and the Desirability of Combining Gene Trees. Taxon 1992, 41:3-10.

8. Ragan MA: Phylogenetic Inference Based on Matrix Representation of Trees. Mol Phylogenet Evol 1992, 1:53-58.

9. Rodrigo AG: On Combining Cladograms. Taxon 1996, 45:267-274.

10. Semple C, Steel M: A supertree method for rooted trees. Discr Appl Math 2000, 105:147-158.

11. Page RDM: Modified Mincut Supertrees. Proceedings of the 2nd Workshop on Algorithms in Bioinformatics (WABI 2002), Volume 2452 of Lecture Notes in Computer Science New York: Springer; 2002, 537-551.

12. Lin HT, Burleigh JG, Eulenstein O: Triplet supertree heuristics for the tree of life. BMC Bioinformatics 2009, 10(Suppl 1):S8.

13. Huson DH, Dezulian T, Klöpper T, Steel MA: Phylogenetic Super-networks from Partial Trees. Proceedings of the 4th Workshop on Algorithms in Bioinformatics (WABI 2004), Volume 3240 of Lecture Notes in Computer Science New York: Springer; 2004, 388-399.

14. Holland B, Conner G, Huber K, Moulton V: Imputing Supertrees and Supernetworks from Quartets. Syst Biol 2007, 56:57-67.

15. Ross HA, Rodrigo AG: An assessment of matrix representation with compatibility in supertree reconstruction. In Phylogenetic Supertrees: Combining Information to Reveal the Tree of Life. Edited by: Bininda-Emonds ORP. Dordrecht, The Netherlands: Kluwer Academic; 2004:35-63.

16. Meacham CA, Estabrook GF: Compatibility Methods in Systematics. Ann Rev Ecol Syst 1985, 16:431-446.

17. Bryant D: A Classification of Consensus Methods for Phylogenetics. In Bioconsensus. Edited by: Janowitz M, Lapointe FJ, McMorris FR, Mirkin B, Roberts FS. Providence, Rhode Island: DIMACS series in discrete mathematics and theoretical computer science. American Mathematical Society; 2003:163-184.

18. Dong J, Fernández-Baca D, McMorris FR, Powers RC: Majority-rule(+) consensus trees. Math Biosci 2010, 228:10-5.

19. Margush T, McMorris FR: Consensus n-trees. Bull Math Biol 1981, 43:239-244.

20. Robinson DF, Foulds LR: Comparison of Phylogenetic Trees. Math Biosci 1981, 53:131-147.

21. Wilkinson M, Cotton JA, Lapointe FJ, Pisani D: Properties of Supertree Methods in the Consensus Setting. Syst Biol 2007, 56(2):330-337.

22. Wilkinson M, Cotton JA, Creevey C, Eulenstein O, Harris SR, Lapointe FJ, Levasseur C, Mclnerney JO, Pisani D, Thorley JL: The Shape of Supertrees to Come: Tree Shape Related Properties of Fourteen Supertree Methods. Syst Biol 2005, 54(3):419-431.

23. Thorley $J \mathrm{~L}$, Wilkinson M: A view of supertree methods. In Bioconsensus. Edited by: Janowitz M, Lapointe FJ, McMorris FR, Mirkin B, Roberts FS. Providence, Rhode Island.: DIMACS series in discrete mathematics and theoretical computer science. American Mathematical Society; 2003:185-193.

24. Phillips CA, Warnow TJ: The asymmetric median tree - A new model for building consensus trees. Discr Appl Math 1996, 71:311-335.

25. Goloboff PA: Minority rule supertrees? MRP, Compatibility, and Minimum Flip may display the least frequent groups. Cladistics 2005, 21(3):282-294.

26. Cotton JA, Wilkinson M: Majority-Rule Supertrees. Syst Biol 2007, 56(3):445-452.

27. Dong J, Fernández-Baca D: Properties of Majority-Rule Supertrees. Syst Biol 2009, 58(3):360-367.

28. Dong J, Fernández-Baca D, McMorris FR: Constructing majority-rule supertrees. Algorithms Mol Biol 2010, 5:2.

29. Bansal MS, Burleigh JG, Eulenstein O, Fernández-Baca D: Robinson-Foulds Supertrees. Algorithms Mol Biol 2010, 5:18.

30. Swofford DL: PAUP*: Phylogenetic Analysis Using Parsimony (* and Other Methods). Version 4 Sinauer Associates, Sunderland, Massachusetts; 2002.

31. Yule GU: A Mathematical Theory of Evolution, Based on the Conclusions of Dr. J. C. Willis, F.R.S. Phil Trans R Soc B 1924, 213:21-87.

32. Sanderson MJ, Ané C, Eulenstein O, Fernandez-Baca D, Kim J, McMahon MM, Piaggio-Talice R: Fragmentation of large data sets in phylogenetic analysis. In Reconstructing evolution: new mathematical and computational advances. Edited by: Gascuel O, Steel MA. Oxford: Oxford University Press; 2007:199-216.

33. Sanderson MJ, McMahon MM, Steel M: Phylogenomics with incomplete taxon coverage: the limits to inference. BMC Evol Biol 2010, 10:155.

34. Waterman MS, Smith TF: On the Similarity of Dendrograms. J Theor Biol 1978, 73:789-800. 
35. Kupczok A, Schmidt $H$, von Haeseler A: Accuracy of phylogeny reconstruction methods combining overlapping gene data sets. Algorithms Mol Biol 2010, 5:37.

36. Creevey CJ, Mclnerney JO: Clann: investigating phylogenetic information through supertree analyses. Bioinformatics 2005, 21(3):390-392.

37. Supertree Estimation Datasets. [http://www.cs.utexas.edu/ phylo/datasets/ supertrees.html].

38. Kennedy M, Page RDM: Seabird supertrees: Combining partial estimates of procellariiform phylogeny. AUK 2002, 119:88-108.

39. Beck R, Bininda-Emonds O, Cardillo M, Liu FG, Purvis A: A higher-level MRP supertree of placental mammals. BMC Evol Biol 2006, 6:93.

40. Creevey CJ, Fitzpatrick DA, Philip GK, Kinsella RJ, O'Connell MJ, Pentony MM Travers SA, Wilkinson M: Does a tree-like phylogeny only exist at the tips in the prokaryotes? Proc R Soc Lond: Biol Sci 2004, 271:2551-2558[http:// bioinf.nuim.ie/supplementary/royalsoc04/].

41. Wu D, Hugenholtz P, Mavromatis K, Pukall R, Dalin E, Ivanova NN, Kunin V, Goodwin L, Wu M, Tindall BJ, Hooper SD, Pati A, Lykidis A, Spring S, Anderson IJ, D'haeseleer P, Zemla A, Singer M, Lapidus A, Nolan M, Copeland A, Han C, Chen F, Cheng JF, Lucas S, Kerfeld C, Lang E, Gronow S, Chain P, Bruce D, Rubin EM, Kyrpides NC, Klenk HP, Eisen JA: A phylogeny-driven genomic encyclopaedia of Bacteria and Archaea. Nature 2009, 462(7276):1056-60.

42. Doolittle WF, Bapteste E: Pattern pluralism and the Tree of Life hypothesis. Proc Natl Acad Sci USA 2007, 104(7):2043-9.

43. Galtier N, Daubin V: Dealing with incongruence in phylogenomic analyses. Philos Trans R Soc Lond B Biol Sci 2008, 363(1512):4023-9.

44. Bapteste E, O'Malley Ma, Beiko RG, Ereshefsky M, Gogarten JP, FranklinHall L, Lapointe FJ, Dupré J, Dagan T, Boucher Y, Martin W: Prokaryotic evolution and the tree of life are two different things. Biol Direct 2009, 4:34.

45. Puigbò P, Wolf YI, Koonin EV: Search for a 'Tree of Life' in the thicket of the phylogenetic forest. J Biol 2009, 8(6):59.

46. Steel M, Rodrigo A: Maximum likelihood supertrees. Syst Biol 2008, 57(2):243-250

47. Kupczok A: Consequences of different null models on the tree shape bias of supertree methods. Syst Biol 2011, 60(2):218-225.

48. Semple C, Steel M: Phylogenetics, Volume 24 of Oxford Lecture Series in Mathematics and Its Applications Oxford, UK: Oxford University Press; 2003.

49. Wilkinson M, Pisani D, Cotton JA, Corfe I: Measuring Support and Finding Unsupported Relationships in Supertrees. Syst Biol 2005, 54(5):823-831.

doi:10.1186/1471-2148-11-205

Cite this article as: Kupczok: Split-based computation of majority-rule supertrees. BMC Evolutionary Biology 2011 11:205.

\section{Submit your next manuscript to BioMed Central and take full advantage of:}

- Convenient online submission

- Thorough peer review

- No space constraints or color figure charges

- Immediate publication on acceptance

- Inclusion in PubMed, CAS, Scopus and Google Scholar

- Research which is freely available for redistribution

Submit your manuscript at www.biomedcentral.com/submit
Biomed Central 